\title{
Correction to: A review of pebble flow study for pebble bed high temperature gas-cooled reactor
}

\author{
Shengyao Jiang', Jiyuan $\mathrm{Tu}^{1,2}$, Xingtuan Yang', Nan Gui' $(\varangle)$ \\ 1. Institute of Nuclear and New Energy Technology, Collaborative Innovation Center of Advanced Nuclear Energy Technology, Key Laboratory \\ of Advanced Reactor Engineering and Safety of Ministry of Education, Tsinghua University, Beijing 100084, China \\ 2. School of Engineering, RMIT University, Melbourne, VIC 3083, Australia
}

(c) The Author(s) 2020, Corrected Publication November 2020

Correction to:

Shengyao Jiang, Jiyuan Tu, Xingtuan Yang, Nan Gui

A review of pebble flow study for pebble bed high temperature gas-cooled reactor

Experimental and Computational Multiphase Flow 2019, 1(3): 159-176

https://doi.org/10.1007/s42757-019-0006-1

The article "A review of pebble flow study for pebble bed high temperature gas-cooled reactor" written by Shengyao Jiang, Jiyuan Tu, Xingtuan Yang, and Nan Gui, was originally published electronically on the publisher's internet portal (currently SpringerLink) on 11 June 2019 without open access. After publication in Volume 1, Issue 3, page 159-176, the author(s) decided to opt for Open Choice and to make the article an open access publication. Therefore, the copyright of the article has been changed to $\odot$ The Author(s) 2020 and the article is forthwith distributed under the terms of the Creative Commons Attribution 4.0 International License (http://creativecommons.org/licenses/by/4.0/), which permits use, duplication, adaptation, distribution and reproduction in any medium or format, as long as you give appropriate credit to the original author(s) and the source, provide a link to the Creative Commons license, and indicate if changes were made.

The original article has been corrected and can be found at https://doi.org/10.1007/s42757-019-0006-1.

$\triangle$ guinan@mail.tsinghua.edu.cn

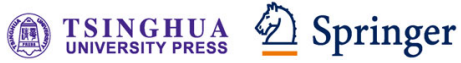

